PROCEEDINGS OF THE

AMERICAN MATHEMATICAL SOCIETY

Volume 127, Number 12, Pages 3495-3502

S 0002-9939(99)04962-X

Article electronically published on May 13, 1999

\title{
CATENARITY IN MODULE-FINITE ALGEBRAS
}

\author{
SHIRO GOTO AND KENJI NISHIDA
}

(Communicated by Ken Goodearl)

\begin{abstract}
The main theorem says that any module-finite (but not necessarily commutative) algebra $\Lambda$ over a commutative Noetherian universally catenary ring $R$ is catenary. Hence the ring $\Lambda$ is catenary if $R$ is Cohen-Macaulay. When $R$ is local and $\Lambda$ is a Cohen-Macaulay $R$-module, we have that $\Lambda$ is a catenary ring, $\operatorname{dim} \Lambda=\operatorname{dim} \Lambda / Q+\operatorname{ht}_{\Lambda} Q$ for any $Q \in \operatorname{Spec} \Lambda$, and the equality $n=\mathrm{ht}_{\Lambda} Q-\mathrm{ht}_{\Lambda} P$ holds true for any pair $P \subseteq Q$ of prime ideals in $\Lambda$ and for any saturated chain $P=P_{0} \subset P_{1} \subset \cdots \subset P_{n}=Q$ of prime ideals between $P$ and $Q$.
\end{abstract}

\section{INTRODUCTION}

Let $R$ be a commutative Noetherian ring and let $\Lambda$ be an $R$-algebra which is finitely generated as an $R$-module. Here we don't assume $\Lambda$ to be a commutative ring. The purpose of this article is to prove the following

Theorem 1.1. Any module-finite $R$-algebra $\Lambda$ is catenary if $R$ is universally catenary.

Before going ahead, let us recall the definition of catenarity and universal catenarity ([Ma, p. 84]). We say that our $\operatorname{ring} \Lambda$ is catenary if for any pair $P \subseteq Q$ of prime ideals in $\Lambda$ and for any saturated chain $P=P_{0} \subset P_{1} \subset \cdots \subset P_{n}=Q$ of prime ideals between $P$ and $Q$, the length $n$ of the chain is independent of its particular choice and hence equal to $\mathrm{ht}_{\Lambda / P} Q / P$, where $\mathrm{ht}_{\Lambda / P} Q / P$ denotes the height of the prime ideal $Q / P$ in $\Lambda / P$. The base $\operatorname{ring} R$ is said to be universally catenary if any finitely generated commutative $R$-algebra is catenary. Hence $R$ is universally catenary if and only if $R / p$ is universally catenary for every prime ideal $p$ in $R$. Naturally, homomorphic images and localizations of universally catenary rings are universally catenary. Our Theorem 1.1 asserts that not necessarily commutative but module-finite $R$-algebras are still catenary if $R$ is universally catenary. The converse is also true in some sense if $R$ is a local integral domain (Corollary 3.4).

Since Cohen-Macaulay rings are necessarily universally catenary, from Theorem 1.1 it follows that

Corollary 1.2. Suppose $R$ is a Cohen-Macaulay ring. Then the ring $\Lambda$ is catenary.

Received by the editors October 27, 1997 and, in revised form, February 24, 1998.

1991 Mathematics Subject Classification. Primary 13E05, 16A18; Secondary 13H10, 16 A33.

The first author was supported by the Grant-in-Aid for Scientific Researches (C).

(C)1999 American Mathematical Society 
In the case where $R$ is local and $\Lambda$ is Cohen-Macaulay as an $R$-module, one can improve Theorem 1.1 to get the following, whose consequences will be discussed in the forthcoming paper [GN].

Corollary 1.3. Assume that $(R, \mathfrak{m})$ is a local ring and $\Lambda$ is a Cohen-Macaulay $R$-module. Then $\Lambda$ is catenary and one has the equality

$$
\operatorname{dim} \Lambda=\operatorname{dim} \Lambda / Q+\operatorname{ht}_{\Lambda} Q
$$

for any prime ideal $Q$ in $\Lambda$ (here $\operatorname{dim} \Lambda / Q$ and $\operatorname{dim} \Lambda$ respectively denote the Krull dimension of the rings $\Lambda / Q$ and $\Lambda)$. The equality $n=\mathrm{ht}_{\Lambda} Q-\mathrm{ht}_{\Lambda} P$ holds true for any pair $P \subseteq Q$ of prime ideals in $\Lambda$ and for any saturated chain $P=P_{0} \subset P_{1} \subset$ $\cdots \subset P_{n}=Q$ of prime ideals between $P$ and $Q$.

The study of catenarity in commutative Noetherian rings dates back to counterexamples of Nagata [N1] in 1956. He also constructed examples of catenary but not universally catenary Noetherian local domains (cf. [N2, p. 203, Example 2]). After Nagata's examples people focused a great deal of their efforts in vain on the problem of whether Noetherian normal local domains are catenary, until Ogoma [O] constructed in 1980 his celebrated counterexamples. Nevertheless their effort was not entirely useless and as an inheritance it has left deep researches on chain conjectures and quasi-unmixed local rings ([R1, R2, R3, R4, Mc], etc.). See Nishimura [Ni] for recent developments.

The study of catenarity in non-commutative Noetherian rings is much steadier. We note here the following two strong results. First, Schelter established the catenarity in Noetherian P.I. algebras finitely generated over central subfields. Subsequently in 1978 he succeeded in deleting the Noetherian assumption from his theorem ([S]). In 1996 Goodearl and Lenagan [GL] showed that certain AuslanderGorenstein and Cohen-Macaulay quantum algebras finitely generated over central subfields are catenary.

The researches $[\mathrm{S}]$ and $[\mathrm{GL}]$ study finitely generated algebras over fields, while we are going to explore module-finite algebras over commutative Noetherian rings. As we shall eventually show in the present paper, comparing with [S] and [GL], our assumption that $\Lambda$ is a module-finite algebra over commutative Noetherian rings $R$ makes the problem simpler and the proof easier. Actually in 1983 Brown, Hajarnavis, and MacEacharn [BHM, 5.2 Theorem] already tried to give a proof of our Corollary 1.3. However it should be mentioned that their argument contains a serious gap.

The proof of Theorem 1.1 will be given in Section 3. In Section 2 we will summarize some basic results on $\Lambda$ that we need to prove Theorem 1.1.

In what follows, let $R$ be a commutative Noetherian ring. Let $\Lambda$ be an $R$-algebra with $f: R \rightarrow \Lambda$ the structure map. The $R$-algebra $\Lambda$ is throughout assumed to be finitely generated as an $R$-module. We denote by $\operatorname{Spec} \Lambda, \operatorname{Min} \Lambda$, and $\operatorname{Max} \Lambda$ the set of prime ideals, the set of minimal prime ideals, and the set of maximal ideals in $\Lambda$, respectively. Unless otherwise specified, all $\Lambda$-modules stand for left $\Lambda$-modules.

\section{Some Basic Results And notation}

The purpose of this section is to summarize some basic results on prime ideals in $\Lambda$ that we shall freely use in Section 3 . We begin with the following, in which for each ideal $I$ in $\Lambda$ let $I \cap R$ denote the inverse image $f^{-1}(I)$ of $I$. 
Lemma 2.1 ([MR, Chapter 10]). Suppose that the structure map $f: R \rightarrow \Lambda$ is injective. Then the following assertions hold true.

(1) (Lying-over) For any prime ideal $p$ in $R$ there is a prime ideal $P$ in $\Lambda$ with $p=P \cap R$.

(2) (Going-up) Let $p \subseteq q$ be prime ideals in $R$ and let $P \in \operatorname{Spec} \Lambda$ with $p=P \cap R$. Then there is a prime ideal $Q$ in $\Lambda$ such that $P \subseteq Q$ and $q=Q \cap R$.

(3) (Incomparability) Let $P \subseteq Q$ be prime ideals in $\Lambda$. Then $P=Q$ if and only if $P \cap R=Q \cap R$.

(4) Let $P \in \operatorname{Spec} \Lambda$. Then $P \in \operatorname{Max} \Lambda$ if and only if $P \cap R \in \operatorname{Max} R$.

(5) For each $P \in$ Min $\Lambda$ the prime ideal $p=P \cap R$ in $R$ consists of zerodivisors for $\Lambda$.

(6) $\operatorname{dim} \Lambda=\operatorname{dim} R=\operatorname{dim}_{R} \Lambda$, where $\operatorname{dim}_{R} \Lambda$ denotes the Krull dimension of $\Lambda$ as an $R$-module.

(7) For each $P \in \operatorname{Spec} \Lambda$ we have $\operatorname{ht}_{\Lambda} p=\mathrm{ht}_{\Lambda_{p}} P \Lambda_{p} \leq \operatorname{dim} R_{p}$, where $p=P \cap R$. In particular $\mathrm{ht}_{\Lambda} P$ is necessarily finite.

In the case where $(R, \mathfrak{m})$ is a local ring, for each finitely generated $R$-module $M$ we put

$$
\operatorname{depth}_{R} M=\inf \left\{n \in \mathbf{Z} \mid \operatorname{Ext}_{R}^{n}(R / \mathfrak{m}, M) \neq(0)\right\}
$$

and call it the depth of $M$. This invariant $\operatorname{depth}_{R} M$ equals the length of maximal $M$-regular sequences contained in $\mathfrak{m}$. See $[\mathrm{BH}]$ and $[\mathrm{Ma}]$ for detailed investigations about it.

Corollary 2.2. Assume that $(R, \mathfrak{m})$ is a local ring and $\operatorname{Max} \Lambda \cap \operatorname{Min} \Lambda \neq \varnothing$. Then the following assertions hold true.

(1) $\operatorname{depth}_{R} \Lambda=0$.

(2) $\operatorname{dim} R=0$ if $\Lambda$ is a free $R$-module.

Proof. Let $P \in \operatorname{Max} \Lambda \cap \operatorname{Min} \Lambda$. Then because $P \in \operatorname{Max} \Lambda$, we have $\mathfrak{m}=P \cap R$ (see 2.1 (4)), whence by 2.1 (5) the ideal $\mathfrak{m}$ consists of zerodivisors for $\Lambda$ since $P \in \operatorname{Min} \Lambda$. Thus $\operatorname{depth}_{R} \Lambda=0$. To see assertion (2) let $p \in \operatorname{Spec} R$. Then since $P \supseteq p \Lambda$, we have $P / p \Lambda \in \operatorname{Max} \Lambda / p \Lambda \cap \operatorname{Min} \Lambda / p \Lambda$. Hence by (1) $\operatorname{depth}_{R / p} \Lambda / p \Lambda=0$, which yields $\operatorname{depth}_{R / p} R / p=0$, because $\Lambda / p \Lambda$ is a free $R / p$-module. Thus $\operatorname{dim} R / p=0$ since $R / p$ is an integral domain, so that we have $\mathfrak{m}=p$. Hence $\operatorname{dim} R=0$.

Lemma 2.3. For each $Q \in \operatorname{Spec} \Lambda$ one has the equality $\operatorname{ht}_{\Lambda} Q=k$, where $k=$ $\inf \left\{n \geq 0 \mid \exists t_{1}, t_{2}, \ldots, t_{n} \in Q \cap R\right.$ such that $Q$ is a minimal prime ideal of $\left.\left(t_{1}, t_{2}, \ldots, t_{n}\right) \Lambda\right\}$.

Proof. We may assume that the structure map $f: R \rightarrow \Lambda$ is injective. Let $h=$ $\operatorname{ht}_{\Lambda} Q$. To check $h \geq k$, we may assume that $h>0$ and our inequality holds true for prime ideals with height at most $h-1$. Let $\mathbf{F}$ be the set of minimal prime ideals $P$ of $\Lambda$ contained in $Q$. Then since $\mathbf{F}$ is a finite set ([MR, 2.2.17]) and since any $P \in \mathbf{F}$ is strictly contained in $Q$, by 2.1 (3) and [AM, 1.11] we see the ideal $Q \cap R$ is not contained in $\bigcup_{P \in \mathbf{F}} P \cap R$. Let $t=t_{1} \in Q \cap R$ with $t \notin \bigcup_{P \in \mathbf{F}} P \cap R$. Then since $\mathrm{ht}_{\Lambda / t \Lambda} Q / t \Lambda \leq h-1$ for the choice of $t$, by the hypothesis on $h$ we may choose elements $t_{2}, t_{3}, \ldots, t_{h}$ of $Q \cap R$ so that $Q$ is a minimal prime ideal of $\left(t_{1}, t_{2}, \ldots, t_{h}\right) \Lambda$. Thus $h \geq k$ and so we get $h_{\Lambda} Q=k$, because $h \leq k$ by [MR, 4.1.13].

In the case where $(R, \mathfrak{m})$ is a local ring, we denote by $R^{*}$ the $\mathfrak{m}$-adic completion of $R$. Recall that $R^{*}$ is a faithfully flat extension of $R$, which is a Noetherian local 
ring with maximal ideal $\mathfrak{m}^{*}=\mathfrak{m} R^{*}$ and $\operatorname{dim} R^{*}=\operatorname{dim} R$. We put $M^{*}=R^{*} \otimes_{R} M$ for each $R$-module $M$.

Corollary 2.4. (1) Suppose $(R, \mathfrak{m})$ is a local ring and let $Q \in \operatorname{Max} \Lambda$. Then $Q^{*} \in$ $\operatorname{Max} \Lambda^{*}$ and $\mathrm{ht}_{\Lambda^{*}} Q^{*}=\mathrm{ht}_{\Lambda} Q$.

(2) Let $Q \in \operatorname{Spec} \Lambda$ and let $t \in Q \cap R$ be a nonzerodivisor for $\Lambda$. Then $\operatorname{ht}_{\Lambda} Q=$ $\mathrm{ht}_{\Lambda / t \Lambda} Q / t \Lambda+1$. Consequently, if $t$ is an element in the Jacobson radical $J(R)$ of $R$ and if $t$ is a nonzerodivisor for $\Lambda$, the equality $\operatorname{dim} \Lambda=\operatorname{dim} \Lambda / t \Lambda+1$ holds true.

Proof. (1) Since $Q \supseteq \mathfrak{m} \Lambda$ and $R^{*} \otimes_{R} R / \mathfrak{m} \cong R / \mathfrak{m}$, we have the isomorphism $\Lambda^{*} / Q^{*}=R^{*} \otimes_{R} \Lambda / Q \cong \Lambda / Q$ and so certainly $Q^{*} \in \operatorname{Max} \Lambda^{*}$. We put $h=\operatorname{ht}_{\Lambda} Q$. The inequality ht $\Lambda_{\Lambda^{*}} Q^{*} \leq h$ follows from 2.3. In fact, choose $t_{1}, t_{2}, \ldots, t_{h} \in Q \cap R$ so that $Q$ is minimal over $I \Lambda$, where $I=\left(t_{1}, t_{2}, \ldots, t_{h}\right) R$. It suffices to see $Q^{*}$ is minimal over $I \Lambda^{*}$ too. Let $P \in \operatorname{Spec} \Lambda^{*}$ with $Q^{*} \supseteq P \supseteq I \Lambda^{*}$. Then clearly $Q=Q^{*} \cap \Lambda \supseteq P \cap \Lambda \supseteq I \Lambda$. Since $P \cap \Lambda \in \operatorname{Spec} \Lambda$ and since $Q$ is minimal over $I \Lambda$, we have $Q=P \cap \Lambda$ whence $Q^{*}=Q \Lambda^{*} \subseteq P$. Thus $Q^{*}$ is minimal over $I \Lambda^{*}$ and by $2.3 \mathrm{ht}_{\Lambda^{*}} Q^{*} \leq h$. Let us check that $\mathrm{ht}_{\Lambda^{*}} Q^{*} \geq h$ by induction on $h$. We may assume that $h>0$ and our inequality holds true for $h-1$. Let $P_{0} \subset P_{1} \subset \cdots \subset P_{h}=Q$ be a saturated chain of prime ideals in $\Lambda$. Then because $\mathrm{ht}_{\Lambda / P_{1}} Q / P_{1}=h-1$, by the hypothesis on $h$ we see that $\mathrm{ht}_{\Lambda^{*} / P_{1}^{*}} Q^{*} / P_{1}^{*} \geq h-1$. Choose $p \in \operatorname{Spec} \Lambda^{*}$ such that $Q^{*} \supseteq p \supseteq P_{1}^{*}$ and $\mathrm{ht}_{\Lambda^{*} / p} Q^{*} / p=\mathrm{ht}_{\Lambda^{*} / P_{1}^{*}} Q^{*} / P_{1}^{*} \geq h-1$. It is enough to show $p \notin \operatorname{Min} \Lambda^{*}$. Let us choose $t \in P_{1} \cap R$ so that $t \notin P_{0} \cap R$ (by 2.1 (3) this choice is possible). Then since $t$ is a nonzerodivisor for $\Lambda / P_{0}$, the element $t$ must be a nonzerodivisor for $\Lambda^{*} / P_{0}^{*}$ too (recall $R^{*}$ is $R$-flat). Therefore if $p$ were a minimal prime ideal in $\Lambda^{*}$, then so is the prime ideal $p / P_{0}^{*}$ in the ring $\Lambda^{*} / P_{0}^{*}$ and hence by 2.1 (5) the ideal $p \cap R^{*}$ consists of zerodivisors for $\Lambda^{*} / P_{0}^{*}$. This is impossible because $t \in p \cap R^{*}$ and $t$ is a nonzerodivisor for $\Lambda^{*} / P_{0}^{*}$. Hence $p \notin \operatorname{Min} \Lambda^{*}$.

(2) By 2.3 we get $h_{\Lambda} Q \leq \mathrm{ht}_{\Lambda / t \Lambda} Q / t \Lambda+1$. The opposite inequality $\mathrm{ht}_{\Lambda} Q \geq$ $\operatorname{ht}_{\Lambda / t \Lambda} Q / t \Lambda+1$ follows from the fact that for any $P \in \operatorname{Min} \Lambda$ the ideal $t \Lambda$ is not contained in $P$ (cf. $2.1(5)$ ). The second assertion is now clear because $t \in Q \cap R$ for all $Q \in \operatorname{Max} \Lambda$ (cf. 2.1 (4)).

Suppose that $R$ is a local ring. Then we say that $R$ is quasi-unmixed if $\operatorname{dim} R^{*} / p$ $=\operatorname{dim} R$ for all $p \in \operatorname{Min} R^{*}$. The next epoch-making characterization is due to Ratliff [R2, Theorem 3.6].

Proposition $2.5([\mathrm{R} 2])$. Suppose that $R$ is a local domain. Then the following two conditions are equivalent.

(1) $R$ is universally catenary.

(2) $R$ is quasi-unmixed.

When this is the case, the ring $R / p$ is also quasi-unmixed and one has the equality $\operatorname{dim} R / p+\operatorname{dim} R_{p}=\operatorname{dim} R$ for all $p \in \operatorname{Spec} R$.

We close this section with a few remarks on canonical modules. Let $R$ be a local ring and assume that $R$ is a homomorphic image of a Gorenstein local ring $S$. Then we put

$$
K_{R}=\operatorname{Ext}_{S}^{n}(R, S) \quad(n=\operatorname{dim} S-\operatorname{dim} R)
$$

and call it the canonical module of $R$. Properties of canonical modules are closely explored in $[\mathrm{HK}]$ and $[\mathrm{BH}]$. Here let us pick up two of them, which we will need to prove Theorem 1.1. We indicate a sketch of the proof for assertion (2), since we have no direct reference for it. 
Lemma 2.6. (1) $K_{\left(R_{p}\right)} \cong\left(K_{R}\right)_{p}$ for any $p \in \operatorname{Supp}_{R} K_{R}$.

(2) Suppose that $\operatorname{dim} R \geq 2$ and let $a_{1}, a_{2}$ be a subsystem of parameters for $R$. Then the sequence $a_{1}, a_{2}$ is $K_{R}$-regular.

Proof. (1) See [HK, Korollar 5.25] or [A, Corollary 4.3].

(2) Write $R=S / I$ with an ideal $I$ in $S$. Then $I$ contains an $S$-regular sequence $f_{1}, f_{2}, \ldots, f_{n}$ of length $n$. Passing to the ring $S /\left(f_{1}, f_{2}, \ldots, f_{n}\right) S$, we may assume $n=0$ whence $K_{R}=\operatorname{Hom}_{S}(R, S)$. Choose an $S$-regular sequence $b_{1}, b_{2}$ so that $a_{1}=b_{1} \bmod I$ and $a_{2}=b_{2} \bmod I$. (This choice is possible. See [K, Theorem 124].) That the sequence $a_{1}, a_{2}$ is $K_{R}$-regular now follows, since $K_{R}=\operatorname{Hom}_{S}(R, S)$ and the sequence $b_{1}, b_{2}$ is $S$-regular.

\section{Proofs of Theorem 1.1 And Corollary 1.3}

The purpose of this section is to prove Theorem 1.1 and Corollary 1.3. We begin with the following.

Lemma 3.1. Suppose that $(R, \mathfrak{m})$ is a local ring and that $R$ is a homomorphic image of a Gorenstein local ring. Let $P \in \operatorname{Spec} \Lambda$ and put $p=P \cap R, \Gamma=\Lambda / P$. Then if $\operatorname{dim} \Gamma \geq 2$, there exist a short exact sequence

$$
0 \rightarrow \Gamma \stackrel{\alpha}{\longrightarrow} X \stackrel{\beta}{\rightarrow} Y \rightarrow 0
$$

of finitely generated $\Gamma$-modules and an element $t \in \mathfrak{m} \backslash p$ satisfying the following conditions.

(1) $\operatorname{depth}_{R} X \geq 2$.

(2) $t$ is $X$-regular and $t Y=(0)$.

Proof. Let $K=K_{R / p}$ be the canonical module of $R / p$. We put

$$
X=\operatorname{Hom}_{R / p}\left(\operatorname{Hom}_{R / p}(\Gamma, K), K\right)
$$

and let $\alpha: \Gamma \rightarrow X$ denote the canonical homomorphism of $\Gamma$-modules. Then $X$ is finitely generated and $\operatorname{depth}_{R} X \geq 2$ by 2.6 (2) because $\operatorname{dim} R / p=\operatorname{dim} \Gamma \geq 2$ by 2.1 (6). Since $K_{p} \cong K_{\left(R_{p} / p R_{p}\right)}=R_{p} / p R_{p}$ by 2.6 (1), we see the homomorphism $R_{p} \otimes_{R} \alpha$ is an isomorphism. Hence $\alpha$ is a monomorphism because the $R / p$-module $\Gamma$ is torsionfree. As $Y_{p}=(0)$ where $Y=\operatorname{Coker} \alpha$, we have $t Y=(0)$ for some $t \in \mathfrak{m} \backslash p$. The element $t$ is $X$-regular since it is $R / p$-regular (cf. 2.6 (2)).

The next result is the key to our proof.

Proposition 3.2. Let $(R, \mathfrak{m})$ be a universally catenary local ring and assume that $\Lambda$ is a prime ring. Then $\operatorname{dim} \Lambda=1$ if $\Lambda$ contains a maximal ideal $Q$ of $\mathrm{ht}_{\Lambda} Q=1$.

Proof. We may assume that $f: R \rightarrow \Lambda$ is injective. Hence $R$ is a local integral domain and $\operatorname{dim} R=\operatorname{dim} \Lambda$ (cf. 2.1 (6)). First we consider the case where $R$ is a homomorphic image of a Gorenstein local ring. Assume that $\operatorname{dim} \Lambda \geq 2$. Then by 3.1 we get an embedding $\Lambda \rightarrow X$ of finitely generated $\Lambda$-modules and an element $0 \neq t \in \mathfrak{m}$ satisfying the conditions (1) $\operatorname{depth}_{R} X \geq 2$ and (2) $t$ is $X$-regular with $t X \subseteq \Lambda$. We put $Z=X / t X$ and $\Delta=\operatorname{End}_{\Lambda} Z$. Let $I=(0):{ }_{\Lambda} Z$. Then since $I X \subseteq t X \subseteq \Lambda$ by condition (2) and since $Q \in \operatorname{Max} \Lambda$ by our assumption, we have $I^{2} \subseteq I^{2} X \subseteq t \Lambda \subseteq Q$ whence $t \Lambda \subseteq I \subseteq Q$. Because $t \notin P \cap R$ for any $P \in \operatorname{Min} \Lambda$ by 2.1 (5) (note that $t$ is $\Lambda$-regular) and because ht ${ }_{\Lambda} Q=1$ by our assumption, we 
have $Q / I \in \operatorname{Min} \Lambda / I \cap \operatorname{Max} \Lambda / I$. Hence $\operatorname{depth}_{R} \Lambda / I=0$ by 2.2 (1). On the other hand, from the embedding

$$
\Lambda / I \rightarrow \operatorname{End}_{\Delta} Z
$$

of $R$-algebras induced from the canonical homomorphism $\lambda: \Lambda \rightarrow \operatorname{End}_{\Delta} Z$, we have $\operatorname{depth}_{R} \Lambda / I>0$ because $\operatorname{depth}_{R} Z=\operatorname{depth}_{R} X-1>0$ by condition (1). This is a contradiction.

Now we consider the general case. Let $R^{*}$ denote the m-adic completion of $R$. Then since $Q^{*}=R^{*} \otimes_{R} Q$ is a maximal ideal of $\Lambda^{*}=R^{*} \otimes_{R} \Lambda$ with ht $\Lambda{ }^{*} Q^{*}=$ $\operatorname{ht}_{\Lambda} Q=1$ (cf. $2.4(1)$ ), we get $\operatorname{ht}_{\Lambda^{*} / P} Q^{*} / P=1$ for some $P \in \operatorname{Min} \Lambda^{*}$. Hence $\operatorname{dim} \Lambda^{*} / P=1$ because $R^{*}$ is a homomorphic image of a Gorenstein (in fact, regular) local ring. Let $p=P \cap R^{*}$. Then we have

Claim. (1) $P \cap \Lambda=(0)$.

(2) $p \in \operatorname{Min} R^{*}$.

Proof of Claim. (1) Note that $P \cap \Lambda \in \operatorname{Spec} \Lambda$ and $Q^{*} \cap \Lambda=Q$. Then if $P \cap \Lambda \neq(0)$, we have $Q=P \cap \Lambda$ since $Q \supseteq P \cap \Lambda \neq(0)$ and ht $\Lambda=1$. Hence $Q^{*} \supseteq P$ which is impossible.

(2) By (1) $p \cap R=(0)$ since the map $f: R \rightarrow \Lambda$ is a monomorphism. Let $Q(R)$ denote the quotient field of $R$. Then since $R_{p}^{*} \otimes_{R^{*}} \Lambda^{*} \cong R_{p}^{*} \otimes_{Q(R)}\left(Q(R) \otimes_{R} \Lambda\right)$, we get $\Lambda_{p}^{*}$ is a free $R_{p}^{*}$-module with $P \Lambda^{*} p \in \operatorname{Max} \Lambda_{p}^{*} \cap \operatorname{Min} \Lambda_{p}^{*}(2.1(4),(7))$. Hence $\operatorname{dim} R_{p}^{*}=0$ by $2.2(2)$.

By Claim (2) and 2.5 we have $\operatorname{dim} R=\operatorname{dim} R^{*} / p=\operatorname{dim} \Lambda^{*} / P=1$. Hence $\operatorname{dim} \Lambda=1$ since $\operatorname{dim} \Lambda=\operatorname{dim} R$.

We now come to the following

Theorem 3.3. Suppose that $R$ is a universally catenary local ring and $\Lambda$ is a prime ring. Then the equality

$$
\operatorname{dim} \Lambda=\operatorname{dim} \Lambda / Q+\mathrm{ht}_{\Lambda} Q
$$

holds true for all $Q \in \operatorname{Spec} \Lambda$.

Proof. We may assume $f: R \rightarrow \Lambda$ is injective. We first consider the case where $Q \in \operatorname{Max} \Lambda$. Let $h=\mathrm{ht}_{\Lambda} Q$. By 3.2 we may assume that $h \geq 2$ and our assertion holds true for $h-1$. Let $(0)=P_{0} \subset P_{1} \subset \cdots \subset P_{h}=Q$ be a saturated chain of prime ideals in $\Lambda$. Let $p=P_{h-1} \cap R$. Then $\operatorname{dim} \Lambda / P_{h-1}=1$ by 3.2. On the other hand, since $P_{h-1} \Lambda_{p}$ is a maximal ideal in the prime ring $\Lambda_{p}$ and $\operatorname{ht}_{\Lambda_{p}} P_{h-1} \Lambda_{p}=$ $h t_{\Lambda} P_{h-1}=h-1(2.1(7))$, by the hypothesis of induction on $h$ the ring $\Lambda_{p}$ has Krull dimension $h-1$. Consequently, by 2.1 (6) and 2.5 we get $\operatorname{dim} \Lambda=\operatorname{dim} R=$ $\operatorname{dim} R / p+\operatorname{dim} R_{p}=\operatorname{dim} \Lambda / P_{h-1}+\operatorname{dim} \Lambda_{p}=1+(h-1)=h$.

Now let $Q \in \operatorname{Spec} \Lambda$ and put $q=Q \cap R$. Then since $Q \Lambda_{q} \in \operatorname{Max} \Lambda_{q}$, we see $\operatorname{dim} \Lambda_{q}=\mathrm{ht}_{\Lambda_{q}} Q \Lambda_{q}=\mathrm{ht}_{\Lambda} Q$. Hence $\operatorname{dim} R_{q}=\mathrm{ht}_{\Lambda} Q$. Because $\operatorname{dim} \Lambda / Q=\operatorname{dim} R / q$ and $\operatorname{dim} \Lambda=\operatorname{dim} R=\operatorname{dim} R / q+\operatorname{dim} R_{q}$ by 2.5 , we get the equality $\operatorname{dim} \Lambda=$ $\operatorname{dim} \Lambda / Q+\operatorname{ht}_{\Lambda} Q$.

Now we are in a position to prove Theorem 1.1.

Proof of Theorem 1.1. Passing to the ring $\Lambda / P$, we may assume $P=(0)$. Let $p=Q \cap R$. Then after the localization at $p$, we may furthermore assume that $R$ is local and $Q \in \operatorname{Max} \Lambda$. Let $(0)=P_{0} \subset P_{1} \subset \cdots \subset P_{n}=Q$ be a saturated chain of prime ideals in $\Lambda$. We will show $n=\operatorname{dim} \Lambda$ by induction on $n$. By 3.2 we may 
assume that $n \geq 2$ and our assertion is true for $n-1$. Then since $\operatorname{dim} \Lambda / P_{1}=n-1$ and $\operatorname{ht}_{\Lambda} P_{1}=1$, we get $n=\operatorname{dim} \Lambda / P_{1}+1=\operatorname{dim} \Lambda / P_{1}+\mathrm{ht}_{\Lambda} P_{1}=\operatorname{dim} \Lambda$ by 3.3 .

Proof of Corollary 1.3. We may assume $f: R \rightarrow \Lambda$ is injective. Let $d=\operatorname{dim} R$. Let $R^{*}$ denote the $\mathfrak{m}$-adic completion of $R$ and we get the embedding $R^{*} \rightarrow \Lambda^{*}$ of $R^{*}$-modules too. Hence $\operatorname{Ass}_{R^{*}} R^{*} \subseteq \operatorname{Ass}_{R^{*}} \Lambda^{*}$ and we have $\operatorname{dim} R^{*} / p=d$ for all $p \in \operatorname{Ass}_{R^{*}} R^{*}$ (cf. [Ma, p. 107]), because $\Lambda^{*}$ is a Cohen-Macaulay $R^{*}$-module of $\operatorname{dim}_{R^{*}} \Lambda^{*}=d$. Therefore for every $p \in \operatorname{Spec} R$ the local ring $R / p$ is quasi-unmixed ([N2,34.5]), whence by $2.5 R / p$ is universally catenary. Thus $R$ is universally catenary, so that by $1.1 \Lambda$ is catenary.

Let $Q \in \operatorname{Min} \Lambda$ and put $q=Q \cap R$. We will show $\operatorname{dim} \Lambda / Q=d$. The prime ideal $q$ consists of zerodivisors for $\Lambda$ (see 2.1 (5)). Hence $q \subseteq \bigcup_{p \in \operatorname{Ass}_{R} \Lambda} p$ (cf. [Ma, p. 50, Corollary 2]) and we have $q \subseteq p$ for some $p \in \operatorname{Ass}_{R} \Lambda$ (cf. [AM, 1.11]). Note that $\operatorname{dim} R / p=d$ (cf. [Ma, p. 107]), because $\Lambda$ is Cohen-Macaulay and $\operatorname{dim}_{R} \Lambda=d$. And we get $q=p$ since $p \in \operatorname{Min} R$. Hence $\operatorname{dim} R / q=d$ so that by 2.1 (6) we have $\operatorname{dim} \Lambda / Q=d$ for all $Q \in \operatorname{Min} \Lambda$.

Thanks to the universal catenarity in $R$, Theorems 1.1 and 3.3 and this observation readily imply that for any pair $P \subseteq Q$ of prime ideals in $\Lambda$ with $P \in \operatorname{Min} \Lambda$ and $Q \in \operatorname{Max} \Lambda$, saturated chains of prime ideals between $P$ and $Q$ have the same length equal to $d=\operatorname{dim} \Lambda$. Hence the equality

$$
\operatorname{dim} \Lambda=\operatorname{dim} \Lambda / Q+\mathrm{ht}_{\Lambda} Q
$$

holds true for any prime ideal $Q$ in $\Lambda$. We also readily have $n=\mathrm{ht}_{\Lambda} Q-\mathrm{ht}_{\Lambda} P$ for any pair $P \subseteq Q$ of prime ideals and for any saturated chain $P=P_{0} \subset P_{1} \subset \cdots \subset$ $P_{n}=Q$ of prime ideals in $\Lambda$. Thus Corollary 1.3 is proven.

To conclude this paper, we note the following. The equivalence is a direct consequence of Theorems 1.1, 3.3, and [R2, Theorems 3.1 and 3.6].

Corollary 3.4. For a commutative Noetherian local integral domain $R$ the following conditions are equivalent.

(1) $R$ is universally catenary.

(2) $R$ is quasi-unmixed.

(3) Every module-finite prime $R$-algebra $\Lambda$ is catenary and the equality

$$
\operatorname{dim} \Lambda=\operatorname{dim} \Lambda / Q+\mathrm{ht}_{\Lambda} Q
$$

holds true for any $Q \in \operatorname{Spec} \Lambda$.

\section{REFERENCES}

[A] Y. Aoyama, Some basic results on canonical modules, J. Math. Kyoto Univ. 23 (1983), 85-94. MR 84i: 13015

[AM] M. F. Atiyah and I. G. MacDonald, Introduction to commutative algebra, Addison Wesley Publishing Company 1969, Menlo Park, California-London-Don Mills, Ontario. MR 39:4129

[BH] W. Bruns and J. Herzog, Cohen-Macaulay rings, Cambridge Studies in Advanced Mathematics 39, Cambridge University Press, Cambridge, New York, Port Chester, Melbourne, Sydney, 1993. MR 95h:13020

[BHM] A. Brown, R. Hajarnavis and A. B. MacEacharn, Rings of finite global dimension integral over their centers, Comm. Alg. 11 (1) (1983), 67-93. MR 84b:16029

[GL] K. R. Goodearl and T. H. Lenagan, Catenarity in quantum algebras, Journal of Pure and Applied Algebra 111 (1996), 123-142. MR 97e:16054

[GN] S. Goto and K. Nishida, On Gorenstein R-algebras, Preprint, 1997. 
[HK] J. Herzog and E. Kunz (eds.), Der Kanonische Modul eines Cohen-Macaulay-Rings, Lecture Notes in Math., 238, Springer-Verlag, Berlin, Heidelberg, New York, Tokyo, 1971. MR 54:304

[K] I. Kaplansky, Commutative rings, Allyn and Bacon, Boston, 1970. MR 40:7234

[Ma] H. Matsumura, Commutative Algebra (second edition), The Benjamin/Cummings Publishing Company, London, Amsterdam, Don Mills, Ontario, Sydney, Tokyo, 1980. MR 82i: 13003

[Mc] S. McAdam, Asymptotic prime divisors, Lecture Notes in Math., 1023, Springer-Verlag, Berlin, Heidelberg, New York, Tokyo, 1983. MR 85f:13018

[MR] J. C. McConnell and J. C. Robson, Noncommutative Noetherian rings, Wiley-Interscience Publishers, New York, London, Sydney, 1987. MR 89j:16023

[N1] M. Nagata, On the chain problem of prime ideals, Nagoya Math. J. 56 (1956), 51-64. MR 18:8e

[N2] M. Nagata, Local rings, Wiley-Interscience Publishers, New York, London, Sydney, 1962.

[Ni] J. Nishimura, A few examples of local rings, Preprint.

[O] T. Ogoma, Non-catenary pseudo-geometric normal rings, Japan. J. Math. 6 (1980), 147163.

[R1] L. J. Ratliff, Jr., On quasi-unmixed semi-local rings and the altitude formula, Amer. J. Math. 87 (1965), 278-284. MR 31:3448

[R2] L. J. Ratliff, Jr., On quasi-unmixed local domains, the altitude formula, and the chain condition for prime ideals (I), Amer. J. Math. 91 (1969), 508-528. MR 40:136

[R3] L. J. Ratliff, Jr., Characterization of catenary rings, Amer. J. Math. 93 (1971), 1070-1108. MR 45:6804

[R4] L. J. Ratliff, Jr., Chain conjectures in ring theory, Lecture Notes in Math., 647, SpringerVerlag, Berlin, Heidelberg, New York, Tokyo, 1978. MR 80c: 13008

[S] W. Schelter, Non-commutative affine P. I. rings are catenary, J. Alg. 51 (1978), 12-18. MR 58:5772

Department of Mathematics, School of Science and Technology, Meiji University, KAWASAKI 214-71, JAPAN

E-mail address: goto@math.meiji.ac.jp

Department of Mathematics, Faculty of Science, Shinsyu University, Matsumoto, 390-0802 JAPAN

E-mail address: kenisida@math.shinsyu-u.ac.jp 